\title{
Deshonestidad académica en estudiantes de una universidad de Costa Rica, América Central
}

\author{
Jensy Campos-Céspedes y Walter Solano Gutiérrez \\ Universidad Estatal a Distancia, 474-2050 San Pedro Montes de Oca, San José, Costa Rica; jensycampos@gmail.com; wsolano@uned.ac.cr
}

Recibido 19-I-2012 Corregido 14-V-2012 Aceptado 12-VII-2012

\begin{abstract}
Academic Dishonesty in students of a Costa Rican university (Central America). Academic dishonesty exists everywhere and has become a research field in recent years. We studied the Academic Dishonesty, (AD), in a Costa Rican university. The information was gathered through a direct analysis of the disciplinary files and the academic files of the total of students charged for AD during the period from 2002 to 2009. $A D$ is irregular since it experiences abrupt increases and decreases in some periods. It does not present significant differences among schools. A fourth of the student offenders are beneficiaries of a scholarship and carry an average academic load of two subjects, situation that dismisses the idea that an excessive academic load may be linked with AD. Most charged students have a low academic performance. The majority of the $A D$ situations occur in situ, that is during the execution of exams and the cases tend to concentrate during the execution of the second ordinary exam. The modality of carrying and copying material during the exam is the most generalized. With regard to the application of sanctions, $74 \%$ of the cases received a ruling that was unfavorable to their interests from the competent authority. Once they fulfill the sanction established by the competent authorities, $77 \%$ of the students charged for AD continue their university studies at the same institution.
\end{abstract}

\section{KEY WORDS}

Academic dishonesty, academic fraud, UNED-Costa Rica, plagiarism, cyberplagiarism.

\section{RESUMEN}

Se estudió la incidencia de la DA en una universidad de Costa Rica. La información se extrajo mediante el análisis directo a los expedientes disciplinarios y los expedientes académicos de la totalidad de los estudiantes inculpados por DA durante el periodo 2002 al 2009. El comportamiento del fenómeno es irregular pues experimenta incrementos y disminuciones abruptas en algunos periodos. No presenta, entre las escuelas diferencias significativas. Sin embargo, existen algunas asignaturas en las que la incidencia de DA ocurre entre dos a 13 ocasiones. Únicamente una cuarta parte de los estudiantes infractores son beneficiarios de beca y llevan una carga académica promedio de dos asignaturas, situación que descarta la idea de que el exceso de carga académica del estudiantes esté ligada a la DA. Asimismo, la mayoría de los estudiantes inculpados, presentan un bajo rendimiento académico. La mayoría de situaciones de DA ocurren in situ, es decir durante la ejecución de exámenes y los casos tienden concentrarse durante la ejecución del segundo examen ordinario y la modalidad de DA referida a portar y copiar material en el examen es la más generalizada. En cuanto a la aplicación de sanciones, el 74\% de los casos recibió un fallo desfavorable a sus intereses por parte de la autoridad competente. Una vez que cumplen la sanción infringida por las autoridades competentes, el 77\% de estudiantes inculpados por DA continúan sus estudios universitarios en la misma institución.

\section{PALABRAS CLAVE}

Deshonestidad académica, fraude académico, UNED-Costa Rica, plagio, ciberplagio.
La deshonestidad académica (DA) es un fenómeno relativamente poco estudiado en el ámbito educativo costarricense. No obstante, en otras latitudes, los análisis acerca del tema se desarrollan desde la década de los años treinta (Vaamonde \& Omar, 2008, p. 7).

Resultados de algunas investigaciones en el tema, indistintamente del país de origen y enfoque (Comas \&
Sureda, 2007; García \& Machado, s.f.; Sureda et al., 2009; Urbina \& Comas, s.f.; Ordóñez et al., 2006) tienden a asociar la comisión de fraude con: el tipo de trabajo solicitado a los estudiantes; las facilidades que ofrece la internet; el desconocimiento para realizar trabajos académicos; la metodología empleada por los académicos; la presión por la consecución de notas altas; las concepciones de 
evaluación como repetición de información memorizada, difícil y sin sentido.

La complejidad del fenómeno de la DA exige que su estudios se desarrolle desde múltiples perspectivas y con un nivel de profundidad que permita su verdadera comprensión. McCabe \& Treviño (1993), citados por Mejía \& Ordoñez (2004), identifican dos perspectivas distintas para el abordaje de la DA: a. "factores personales individuales $(. .$.$) " y b. "factores institucionales externos al individuo$ (...)" (p. 15). En el estudio se concibe la DA como un fenómeno multidimensional, multicausal y multifactorial. Sin embargo, los resultados que se ofrecen en este artículo se centran en las percepciones que el personal académico de la UNED- Costa Rica presenta respecto del fenómeno.

\section{METODOLOGÍA}

Como técnica de recolección de información, se empleó el análisis directo de dos fuentes: los expedientes disciplinarios (219 casos) y el expediente académico de los casos de DA procesado durante los períodos académicos de 2002-3 al 2009-4. Las variables consideradas fueron: tipología de fraude, carrera, curso, periodo académico, condición socioeconómica del estudiante, medida correctiva aplicada. Se extrajeron, también, de los expedientes académicos de los estudiantes inculpados por DA, los datos referidos a; rendimiento académico, tiempo transcurrido antes de la reinserción a la universidad y la condición de becario. Como garantía de confidencialidad de la información se asignó un número a cada uno de los casos y todos los análisis de los datos se trabajaron en función del número de caso sin utilizar el nombre de los sujetos incluidos.

\section{RESULTADOS}

El 65,5\%, de los inculpados pertenecen al sexo femenino y el $34,7 \%$ al sexo masculino. Este dato debe manejarse de manera ponderada, debido a que porcentualmente el sexo femenino ha sido históricamente superior al masculino en la composición del estudiantado de la universidad.

El 69,7\% de estos sujetos se ubica entre los ámbitos de edad 19 a 36 años; siendo la edad promedio 30,5 años. En cuanto al estado civil, el $48,8 \%$ se manifiestan solteros (as), un $38,8 \%$ casados (as). El grado académico de ingreso a la universidad, en un $99 \%$ es el bachillerato en secundaria y solo un $1 \%$ es el de diplomado o licenciatura en una carrera universitaria.

Los centros universitarios de San José, Heredia, Alajuela y Cartago agrupan el 45,2\% de los casos y presentan periódicamente el mayor número de estudiantes matriculados.
La condición de becario, únicamente se presenta en el $29,2 \%$ de los estudiantes de la muestra. De este porcentaje el $53 \%$ son mujeres y el $47 \%$ hombres. El tipo de beca, mayormente solicitado $(73,43 \%)$ es la categoría B. Esta categoría de beca, según el reglamento respectivo, implica exención del $100 \%$ de la tarifa.

En lo que respecta al número asignaturas matriculadas en el período académico en el que se incurre en la falta, el $82,1 \%$, reporta una carga académica de 2 a 4 asignaturas.

La incidencia de casos de DA, durante los periodos comprendidos en el estudio se comporta de manera discontinua. Entre los años 2002 al 2004, se nota una tendencia creciente de este fenómeno. Luego, desde el 2005 hasta el 2007 se aprecia una disminución progresiva. Para el periodo 2008 hay un incremento desmesurado. No obstante, durante el año 2009 se advierte una recaída. Este último periodo contempla, como se informó anteriormente, únicamente dos cuatrimestres (Cuadro 1).

La DA, de acuerdo con los datos, parece ser un fenómeno generalizado en las Escuelas. La diferencia entre ellas no es en extremo notable. Las dos Escuelas que presentan valores porcentuales más elevados son: la Escuela de Ciencias Sociales y Humanidades (ECSH) con $31,50 \%$ y la Escuela de Ciencias de la Educación ( ECE) con 26,02\%, tal como se observa en el Cuadro 2.

De las 96 asignaturas en las que se reportan situaciones de DA, en 47,91\% de los casos, la frecuencia ocurre entre 2 a 13 ocasiones en algunos cursos. En la ECE en 11 asignaturas la comisión de fraude tiene un promedio de 4,36\%; en la Escuela de Ciencias Exactas y Naturales (ECEN) en 7 asignaturas el fraude se da en un promedio de 4,28\%. Los promedios relativamente más bajos se presentan, respectivamente, en la ECSH con 13 asignaturas (3,53\%) y en la Escuela de Ciencias de la Administración (ECA) con 10 asignaturas (3,8\%). En la ECEN, por su parte, se concentra el menor número de asignaturas (7) y una mayor incidencia de DA con respecto a la cantidad de asignaturas.

La ECA presenta un caso bastante particular: en la asignatura Finanzas I se exponen 13 casos de comisión de fraude. Once de ellos se presentan en un solo periodo académico (2008-3) y siete ocurren en el Centro Universitario de Heredia; los restantes se distribuyen entre el 2005-4 y 2005-5. Por otra parte, en la ECE, la asignatura Didáctica del Lenguaje y Estudios Sociales para Alumnos con Necesidades Especiales también se presentan 11 casos de fraude académico. No obstante, estos se distribuyen en 7 periodos académicos.

La carga académica (número de asignatura que se matrícula por cuatrimestre) implica -entre otros asuntospara el estudiante; disponibilidad de tiempo, esfuerzo mental, expectativas de celeridad y, desde luego, recursos 


\section{CUADRO 1}

Distribución de la comisión de fraude académico según año lectivo.

\begin{tabular}{cc}
\hline Periodo académico & Cantidad de casos (\%) \\
\hline 2002 & 7,30 \\
2003 & 9,58 \\
2004 & 14,15 \\
2005 & 13,24 \\
2006 & 11,87 \\
2007 & 9,58 \\
2008 & 26,02 \\
$2009 *$ & 8,21 \\
Total de casos= 219 & $100 \%$ \\
*se incluyeron solo los casos de 2 cuatrimestres.
\end{tabular}

CUADRO 2

Distribución de la comisión de fraude académico según Escuela.

\begin{tabular}{cc}
\hline Escuela & Cantidad de casos (\%) \\
\hline ECE & 26 \\
ECSH & 31 \\
ECEN & 20 \\
ECA & 22
\end{tabular}

Total de casos $=219$

CUADRO 3

Distribución de carga académica según sexo.

\begin{tabular}{lccc}
\hline \multirow{2}{*}{ Cantidad de asignaturas } & \multicolumn{3}{c}{ Porcentaje } \\
\cline { 2 - 4 } & Mujeres & Hombres & Total \\
\hline Una asignatura & 6 & 4 & 10 \\
Dos asignaturas & 22 & 9 & 31 \\
Tres asignaturas & 18 & 6 & 25 \\
cuatro asignaturas & 15 & 11 & 26 \\
cinco asignatura & 2 & 2 & 4 \\
6 o más asignaturas & 1 & 2 & 4 \\
Total & 65 & 35 & 100 \\
*Se contemplan 218 casos; había información incompleta en un \\
expediente disciplinario.
\end{tabular}

económicos. Se trata de condiciones que de algún modo exigen un compromiso de responsabilidad.

Como se indicó antes, únicamente $29 \%$ de la muestra de estudiantes posee la condición de becario; la mayor parte de ellos disfrutan de la categoría de beca B. Esta categoría demanda una carga académica mínima de dos asignaturas por cuatrimestre. La validación, en la misma categoría, exige la aprobación de ese mínimo. El reglamento de becas de la institución aclara que en caso de estudiantes que no cumplan con la aprobación de las asignaturas mínimas, su condición de becario cambiará a la categoría inmediata inferior.

Indistintamente del sexo, la mayoría de los estudiantes, $31,19 \%$, reportaba una carga académica, en el momento de la infracción, de dos asignaturas; una asignatura, $10,09 \%$; tres asignaturas, $24,77 \%$; cuatro asignaturas, $26,14 \%$; cinco asignaturas, $4,12 \%$ y seis o más asignaturas, 3,66\%. Como se muestra en el Cuadro 3.

La distribución por Escuela de la carga académica de los estudiantes infractores, permite observar que en la ECSH tiende a presentarse la mayor concentración de estudiantes a partir de las diferentes designaciones de carga académica. Excepto en la carga académica de cuatro asignaturas, en que los valores de la ECE, la ECEN y la ECA son un tanto superiores, lo cual se aprecia en el Cuadro 4.

El 55,96\% de los estudiantes inculpados lograron, hasta el periodo académico de su detección, un promedio ponderado inferior a 7 (bajo); 37,61\% correspondió a un promedio ponderado de 7 a 8,5 (regular); únicamente, 6,42 de los inculpados presentó un promedio ponderado de 8,6 a 9,50 (alto). El Cuadro 5 resume los datos descritos.

Por otra parte, el promedio ponderado parece no estar estrictamente asociado con la variable sexo. En este sentido, no se detectó diferencias estadísticamente significativas entre ambos grupos.

Los estudiantes de bajo rendimiento (promedio ponderado inferior a 7) representan $55,96 \%$ de la muestra (122 de 218 sujetos). La mayoría, 35,24\% (77 personas), tiende a matricular una carga académica de dos asignaturas por cuatrimestre. En el caso de los estudiantes del grupo de promedio regular (7 a 8,50) la cifra es $28,04 \%$ (61 individuos). Un caso particular es el de los estudiantes de alto rendimiento $(8,6$ a 9,50$)$ donde de 14 estudiantes $(6,42 \%)$ en esta condición, seis matricularon una carga académica de cuatro asignaturas durante el cuatrimestre respectivo. En todo caso, la mayoría de los estudiantes en esta condición de carga académica $(31,19 \%)$ evidencia un peso académico de dos asignaturas por cuatrimestre (Cuadro 6). 


\section{CUADRO 4}

Distribución de carga académica de los estudiantes inculpados por DA, según Escuela.

\begin{tabular}{lcccc}
\hline Carga académica & ECE & ECSH & ECEN & ECA \\
\hline Una asignatura & 4 & 8 & 2 & 8 \\
Dos asignaturas & 21 & 30 & 10 & 7 \\
Tres asignaturas & 14 & 17 & 12 & 11 \\
Cuatro asignaturas & 15 & 10 & 18 & 14 \\
Cinco asignaturas & 1 & 2 & 2 & 4 \\
Seis o más asignaturas & 2 & 2 & 1 & 3 \\
Total $^{*}$ & 57 & 69 & 45 & 47
\end{tabular}

* Se contemplan 218 casos; había información incompleta en un expediente disciplinario.

CUADRO 5

Promedio ponderado según sexo.

\begin{tabular}{lccc}
\hline \multirow{2}{*}{ Promedio ponderado } & \multicolumn{2}{c}{ Sexo } & N $^{\mathbf{0}}$ \\
\cline { 2 - 3 } & Mujer \% & Hombre \% & Total \\
\hline Bajo (menor de 7) & 61 & 46 & 122 \\
Regular (+ 7 y - 8,5) & 32 & 48 & 82 \\
Alto $(8,6$ a 9,5) & 7 & 5 & 14 \\
Total* & \multicolumn{3}{c}{} \\
* Se contemplan 218 casos; había información incompleta en un \\
expediente disciplinario.
\end{tabular}

CUADRO 7

Distribución de casos analizados con disfrute de beca según rango de edad.

\begin{tabular}{|c|c|}
\hline Edad & Porcentaje \\
\hline Menor de 18 & 0 \\
\hline De 19 a 24 & 26 \\
\hline De 25 a 30 & 33 \\
\hline De 31 a 36 & 22 \\
\hline De 37 a 42 & 7 \\
\hline De 43 a 48 & 3 \\
\hline De 49 a 54 & 9 \\
\hline De 55 a 60 & 0 \\
\hline Total $=58$ & 100 \\
\hline
\end{tabular}

De acuerdo con las cifras presentadas no se evidencia asociación entre el alto número de asignaturas matriculadas y la comisión de DA.

De los 219 casos de estudiantes inculpados de DA, únicamente 64 sujetos presentaron la condición de becarios. El $53,12 \%$ correspondió al sexo femenino y $48,87 \%$ al masculino. El rango de edad 25 a 30 años agrupó, relativamente, el mayor porcentaje, es decir, $32,75 \%$ de dichos individuos (Cuadro 7).

La categoría de beca de más recurrencia es la $\mathrm{B}$, con $73,43 \%$ de asignatarios, seguida de la categoría $C$ con 15,62\% (Cuadro 8).

De la muestra de estudiantes inculpados por DA, $61 \%$ de las supuestas infracciones, ocurren con la aplicación del primero y del segundo ordinario. El mayor valor, 36\%, se alcanza en el segundo examen ordinario.

CUADRO 6

Carga académica según promedio ponderado.

\begin{tabular}{lcccc}
\hline Carga académica & $\begin{array}{c}\text { Bajo } \\
(\%)\end{array}$ & $\begin{array}{c}\text { Regular } \\
(\%)\end{array}$ & $\begin{array}{c}\text { Alto } \\
(\%)\end{array}$ & $\begin{array}{c}\text { Total } \\
(\%)\end{array}$ \\
\hline Una asignatura & 12 & 7 & 7 & 10 \\
Dos asignaturas & 35 & 28 & 14 & 31 \\
Tres asignaturas & 23 & 26 & 36 & 25 \\
Cuatro asignaturas & 23 & 28 & 43 & 26 \\
Cinco asignaturas & 3 & 6 & 0 & 4 \\
Seis o más asignaturas & 3 & 5 & 0 & 4 \\
Total & 56 & 38 & 6 & 100
\end{tabular}

CUADRO 8

Distribución de casos según categoría de beca.

\begin{tabular}{cc}
\hline Categoría & Porcentaje \\
\hline Beca A & 1,5 \\
Beca B & 73 \\
Beca C & 16 \\
Beca D & 8 \\
Beca de estímulo & 1,5 \\
Total= 58 & 100
\end{tabular}


CUADRO 9

Situaciones de DA según tipo de instrumento.

\begin{tabular}{cc}
\hline Instrumento & Porcentaje \\
\hline Primer ordinario & 25 \\
Segundo ordinario & 36 \\
Reposición & 13 \\
Tareas & 14 \\
Suficiencia & 2 \\
TFG & 2 \\
Proyectos $^{*}$ & 8 \\
Total de casos $=219$ & 100
\end{tabular}

* Proyecto se entiende como el ejercicio académico con valor semejante al de una tarea. En algunos casos sustituye un primero o segundo examen ordinario. Generalmente tiene una función práctica en el proceso de evaluación.
La mayoría de los casos de DA analizados corresponden a infracciones cometidas durante el segundo examen ordinario, tal como se puede observar en el Cuadro 9.

La información que presenta el cuadro 10 es particularmente provocativa. ¿Qué factores se conjugan para que el fraude académico en exámenes agrupe más de la tercera parte de los casos, específicamente en el segundo examen ordinario y por qué estos porcentajes son recurrentes en las cuatro Escuelas? En principio, se descarta el azar. Una hipotética explicación debería contemplar las siguientes circunstancias: a) un alto porcentaje de los estudiantes inculpados de fraude académico evidencian un promedio ponderado bajo; b) los exámenes, en la mayoría de los eventos de evaluación, representan el más alto valor porcentual de la calificación final; c) el segundo examen ordinario podría ser una de las últimas oportunidades con que cuenta el estudiante para mejorar la nota final.

La conjugación de estas tres circunstancias podría propiciar en los estudiantes infractores la necesidad de aplicar el criterio de "maximización de utilidades", es decir, valorar los costes y beneficios con el objeto de que mediante el menor esfuerzo obtener los "mejores resultados" $y$, en esta coyuntura, el fraude académico es una tentadora salida dada la premura de la situación (García \& Machado, s. f.).

La distribución del fraude académico por sexo permite observar que la mayoría de las mujeres incluidas en el estudio incurren en esta falta con la aplicación del primer examen ordinario. En el caso de los hombres, ocurre lo contrario: $45 \%$ de ellos incurre en tal falta con la aplicación del segundo ordinario. En cuanto DA cometida en tareas
CUADRO 10

Fraude por sexo según instrumento.

\begin{tabular}{ccc}
\hline Tipo de instrumento & Mujeres (\%) & Hombres (\%) \\
\hline Primer ordinario & 29 & 17 \\
Segundo ordinario & 31 & 45 \\
Reposición & 14 & 12 \\
Tareas & 13 & 17 \\
Suficiencia & 3 & 0 \\
TFG & 2 & 1 \\
Proyectos & 8 & 8
\end{tabular}

y proyectos (18 más 11 respectivamente), 29 de los casos corresponde a mujeres y $25 \%$ de los casos a varones (Cuadro 10).

De acuerdo con los datos, extraídos de los 219 expedientes disciplinarios analizados, el fraude académico cometido en Trabajos Finales de Graduación (TFG) podría estar presentando valores bajos (con respecto a la incidencia de la DA en otros instrumentos de evaluación), indistintamente de la variable sexo. Sin embargo, la DA en los TFG podría estar siendo subregistado en virtud de que no se cuentan con mecanismos ni estrategias sistemáticas para la correcta detección. Asimismo, el desarrollo de las Tecnologías de la Información y Comunicación (TIC) ofrece nuevas posibilidades de uso indebido de la información disponible en la red mundial, tal como lo señalan Comas y Sureda, 2007.

Según la información institucional del comportamiento de la matricula por centro universitario, históricamente estos tienen un patrón estable en cuanto al porcentaje de estudiantes que matriculan. Cuando se relaciona el porcentaje de matrícula que cada centro universitario presenta en un periodo con la cantidad de casos de DA reportados en el mismo lapso, se nota que algunos centros universitarios presentan hasta dos o tres desviaciones estándar del comportamiento que el fenómeno asume para la institución. Eso significa que, proporcionalmente, la incidencia del fenómeno se presenta más en los centros universitarios de Nicoya, Santa Cruz, Limón, Tilarán, Upala y La Reforma (se presentan los centros universitarios en forma creciente) (Cuadro 11). 
CUADRO 11

Distribución de centros universitarios según matrícula y casos de DA durante el periodo en estudio.

\begin{tabular}{|c|c|c|c|c|c|c|c|c|c|c|c|c|}
\hline \multirow{2}{*}{ Centro Universitario } & \multicolumn{9}{|c|}{ Matrícula anual } & \multirow{2}{*}{$\begin{array}{l}\text { Incidencia } \\
\text { detectada }\end{array}$} & \multirow{2}{*}{$\begin{array}{l}\text { Matrícula } \\
\text { promedio }\end{array}$} & \multirow{2}{*}{$\begin{array}{l}\text { Incidencia } \\
\text { por cada mil } \\
\text { estudiantes }\end{array}$} \\
\hline & 2002 & 2003 & 2004 & 2005 & 2006 & 2007 & 2008 & 2009 & 2010 & & & \\
\hline Ciudad Neilly & 246 & 284 & 334 & 314 & 295 & 330 & 358 & 354 & 389 & - & 323 & 0,00 \\
\hline Osa & 91 & 109 & 106 & 89 & 87 & 90 & 81 & 111 & 106 & - & 97 & 0,00 \\
\hline Sarapiquí & 37 & 45 & 91 & 96 & 123 & 126 & 130 & 163 & 189 & - & 111 & 0,00 \\
\hline San Vito & 178 & 182 & 162 & 176 & 181 & 191 & 223 & 284 & 339 & - & 213 & 0,00 \\
\hline Jicaral & 49 & 59 & 53 & 63 & 55 & 64 & 53 & 62 & 74 & - & 59 & 0,00 \\
\hline La Cruz & 59 & 83 & 92 & 114 & 127 & 128 & 149 & 147 & 159 & - & 118 & 0,00 \\
\hline Buenos Aires & 16 & 27 & 26 & 26 & 42 & 54 & 76 & 129 & 232 & - & 70 & 0,00 \\
\hline Monteverde & 61 & 69 & 73 & 75 & 86 & 91 & 95 & 92 & 97 & - & 82 & 0,00 \\
\hline Puerto Jiménez & 15 & 22 & 40 & 33 & 36 & 40 & 50 & 43 & 42 & - & 36 & 0,00 \\
\hline Pavón & 32 & 35 & 38 & 41 & 53 & 78 & 71 & 76 & 80 & - & 56 & 0,00 \\
\hline Talamanca & 2 & 11 & 22 & 25 & 50 & 68 & 71 & 107 & 119 & - & 53 & 0,00 \\
\hline Acosta & 6 & 4 & 4 & 3 & 4 & 7 & 10 & 14 & 86 & - & 15 & 0,00 \\
\hline $\begin{array}{l}\text { Sede Interuniversitaria } \\
\text { Alajuela }\end{array}$ & 27 & 32 & 32 & 32 & 31 & 107 & 160 & 175 & 261 & - & 95 & 0,00 \\
\hline $\begin{array}{l}\text { San Ramón Sede } \\
\text { Interuniversitaria }\end{array}$ & 15 & 16 & 15 & 13 & 13 & 38 & 34 & 25 & 12 & - & 20 & 0,00 \\
\hline $\begin{array}{l}\text { Puntarenas Sede } \\
\text { Interuniversitaria }\end{array}$ & 5 & 7 & 2 & 4 & 5 & 13 & 45 & 27 & 23 & - & 15 & 0,00 \\
\hline $\begin{array}{l}\text { Turrialba Sede } \\
\text { Interuniversitaria }\end{array}$ & 0 & 0 & 0 & 0 & 1 & 2 & 3 & 1 & 4 & - & 1 & 0,00 \\
\hline $\begin{array}{l}\text { Ciudad Neilly Sede } \\
\text { Interuniversitaria }\end{array}$ & 0 & 0 & 0 & 0 & 0 & 0 & 3 & 21 & 17 & - & 5 & 0,00 \\
\hline Limón Carreras conjuntas & 0 & 0 & 0 & 0 & 1 & 0 & 2 & 2 & 10 & - & 2 & 0,00 \\
\hline San Carlos & 1136 & 1117 & 1198 & 1140 & 1197 & 1193 & 1096 & 1103 & 1142 & 3 & 1147 & $2,62^{*}$ \\
\hline Cañas & 347 & 329 & 350 & 343 & 357 & 329 & 335 & 318 & 363 & 1 & 341 & $2,93^{*}$ \\
\hline Guapilés & 559 & 615 & 615 & 650 & 589 & 669 & 684 & 692 & 770 & 2 & 649 & $3,08^{*}$ \\
\hline Desamparados & 532 & 620 & 1201 & 1480 & 1481 & 1589 & 1653 & 1715 & 1768 & 5 & 1338 & $3,74^{*}$ \\
\hline San Isidro & 762 & 774 & 711 & 676 & 658 & 687 & 640 & 702 & 877 & 3 & 721 & $4,16^{*}$ \\
\hline Atenas & 217 & 206 & 191 & 185 & 180 & 188 & 181 & 188 & 199 & 1 & 193 & $5,19^{*}$ \\
\hline San José & 8418 & 8621 & 8148 & 7784 & 7469 & 7500 & 7218 & 7497 & 7637 & 41 & 7810 & $5,25^{*}$ \\
\hline Turrialba & 768 & 805 & 955 & 1061 & 1141 & 1188 & 1166 & 1175 & 1342 & 6 & 1067 & $5,62^{*}$ \\
\hline San Marcos & 251 & 279 & 281 & 311 & 340 & 341 & 344 & 377 & 433 & 2 & 329 & $6,09^{*}$ \\
\hline Palmares & 1808 & 1850 & 1869 & 1875 & 1801 & 1897 & 1819 & 1974 & 2058 & 12 & 1883 & $6,37^{*}$ \\
\hline Alajuela & 2005 & 2038 & 1977 & 1844 & 1774 & 1806 & 1761 & 1841 & 1953 & 14 & 1889 & $7,41^{*}$ \\
\hline Puntarenas & 739 & 655 & 671 & 624 & 613 & 573 & 588 & 612 & 766 & 5 & 649 & $7,70^{*}$ \\
\hline Cartado & 1871 & 1938 & 2036 & 2039 & 2153 & 2166 & 2126 & 2133 & 2285 & 20 & 2083 & $9,60^{*}$ \\
\hline Heredia & 2391 & 2519 & 2585 & 2417 & 2322 & 2352 & 2215 & 2307 & 2460 & 24 & 2396 & $10,01 *$ \\
\hline Siquirres & 228 & 202 & 235 & 252 & 274 & 265 & 271 & 275 & 349 & 3 & 261 & $11,48^{*}$ \\
\hline Puriscal & 627 & 653 & 657 & 650 & 654 & 594 & 567 & 597 & 677 & 8 & 631 & $12,68^{* *}$ \\
\hline Quepos & 142 & 152 & 147 & 136 & 146 & 127 & 156 & 175 & 208 & 2 & 154 & $12,96^{* *}$ \\
\hline Orotina & 255 & 240 & 206 & 171 & 196 & 193 & 195 & 221 & 280 & 3 & 217 & $13,80^{* *}$ \\
\hline Liberia & 520 & 488 & 561 & 565 & 526 & 507 & 504 & 541 & 577 & 9 & 532 & $16,91^{* *}$ \\
\hline Extranjeros & 55 & 68 & 69 & 65 & 56 & 45 & 34 & 31 & 35 & 1 & 51 & $19,65^{* *}$ \\
\hline Nicoya & 380 & 338 & 404 & 331 & 311 & 276 & 260 & 279 & 327 & 8 & 323 & $24,78^{* * *}$ \\
\hline Santa Cruz & 476 & 443 & 468 & 459 & 421 & 374 & 304 & 280 & 317 & 11 & 394 & $27,95^{* * *}$ \\
\hline La Reforna & 35 & 42 & 50 & 50 & 57 & 56 & 61 & 86 & 99 & 2 & 60 & $33,58^{* * *}$ \\
\hline Limón & 433 & 420 & 400 & 387 & 370 & 390 & 377 & 436 & 461 & 14 & 408 & $34,30 * * *$ \\
\hline Tilaran & 97 & 108 & 107 & 127 & 99 & 120 & 108 & 137 & 138 & 4 & 116 & $34,58^{* * *}$ \\
\hline Upala & 192 & 201 & 172 & 169 & 177 & 201 & 226 & 216 & 289 & 9 & 205 & $43,95^{* * * *}$ \\
\hline
\end{tabular}

*una desviación estándar; ${ }^{* *}$ dos desviaciones estándar; ${ }^{* * *}$ tres desviaciones estándar; ${ }^{* * * *}$ cuatro desviaciones estándar 
En cuanto al tipo de DA registrado en los casos analizados, la mayoría (49,3\%) ocurre, aparentemente, in situ; Es decir, durante la aplicación de los instrumentos de evaluación. Un segundo tipo de DA encontrado fue el denominado respuestas idénticas a las brindadas por otro (a) compañero (a); que ocurre en $23,7 \%$ de los casos. El fraude de alteración de instrumentos de evaluación, representa $10,5 \%$. Los otros tipos de DA conjuntan un valor porcentual de $16,4 \%$ (Cuadro 12).

De acuerdo con los datos encontrados, el tipo de DA de copiar en el desarrollo de los exámenes pareciera ser una constante en los contextos educativos universitarios. Sobre este tema específico, Fernández \& Ordóñez (2004) detectaron "que $50 \%$ de los estudiantes ha copiado alguna vez en un examen" (p.18).

\section{CUADRO 12}

Distribución de la falta según tipología.

\begin{tabular}{lc}
\hline \multicolumn{1}{c}{ Tipología } & Porcentaje \\
\hline Portar y copilar la materia en un examen & 49,3 \\
$\begin{array}{l}\text { Respuestas idénticas a las brindadas por } \\
\text { otro(a) compañero(a) }\end{array}$ & 23,7 \\
$\begin{array}{l}\text { Alteración de los instrumentos de evaluación } \\
\text { ya solucionados }\end{array}$ & 10,5 \\
Respuestas idénticas al solucionarlo & 7,3 \\
Plagio & 6,4 \\
Otra & 2,7 \\
Total de casos $=219$ & 100
\end{tabular}

En cuanto al tipo de DA según sexo, no se presenta marcadas diferencias, o sea que no hay valores estadísticamente significativos que demuestren que el sexo determina la tipología de falta. En ambos casos (en hombres y mujeres) el tipo de DA que más ocurre es portar y copiar la materia en un examen tal como se observa en el Cuadro 13.

En cuanto al tipo de DA y su distribución en cada una de las Escuelas, los datos muestras que la modalidad de falta correspondiente a portar y copiar material de un examen, es más frecuente en la Escuela de Ciencias Sociales y Humanidades, en que representa $75 \%$ de los casos en esa tipología. La Escuela de Ciencias de la Administración agrupa $40 \%$ y la Escuela de Ciencias de la Educación acumula $37 \%$.

La otra tipología que acumula un número de casos representativo son las respuestas idénticas al solucionario; en dicha modalidad, la ECE acumula el mayor porcentaje de casos, con $54 \%$ de las situaciones detectadas (Cuadro 14).

De los 216 casos de estudiantes inculpados de DA, 74\% recibió un fallo desfavorable a sus intereses. Únicamente a $26 \%$ se les exoneró de la falta. El patrón de los datos según sexo indica que de las 141 mujeres inculpadas por DA, a $73 \%$ no les favoreció la resolución dictada; asimismo, $26 \%$ fue absuelto de toda responsabilidad. En caso de los hombres, de un total de 75 casos, $73 \%$ recibió un fallo adverso y solo $27 \%$ favorable (Cuadro 15). Por otra parte, en estos datos no se identificó diferencias estadísticamente significativas entre ambos sexos.

CUADRO 13

Distribución de los casos según tipología y sexo.

\begin{tabular}{lccc}
\hline \multicolumn{1}{c}{ Tipología o modalidad de la falta } & \multicolumn{2}{c}{ Sexo } & Total \\
\cline { 2 - 3 } & Mujer (\%) & Hombre (\%) & 49,3 \\
Portar y copiar la materia en un examen & 74,1 & 25,9 & 23,7 \\
Respuestas idénticas a las brindadas por otro(a) compañero (a) & 59,6 & 40,4 & 10,5 \\
Alteración de los instrumentos de evaluación y a solucionados & 60,9 & 39,1 & 7,3 \\
Respuestas idénticas al solucionarlo & 37,5 & 62,5 & 6,4 \\
Plagio & 57,1 & 42,9 & 2,7 \\
Otra & 66,7 & 33,3 & 100
\end{tabular}


CUADRO 14

Distribución de la falta según tipología según Escuela.

\begin{tabular}{lccccc}
\hline \multicolumn{1}{c}{ Falta } & ECE (\%) & ECSH (\%) & ECA (\%) & ECEN (\%) & Total (\%) \\
\hline $\begin{array}{l}\text { Portar y copiar la materia en un examen } \\
\text { Respuestas idénticas a las brindadas por }\end{array}$ & 37 & 75 & 40 & 35 & 108 \\
$\begin{array}{l}\text { otro(a) compañero (a) } \\
\text { Alteración de los instrumentos de }\end{array}$ & 0 & 6 & 10 & 27 & 41 \\
evaluación ya solucionados & 4 & 7 & 15 & 20 & 43 \\
Respuestas idénticas al solucionarlo & 54 & 0 & 27 & 7 & 16 \\
Plagio & 5 & 7 & 8 & 4 & 14 \\
Otra & - & 5 & - & 7 & 6 \\
Total & 100 & 100 & 100 & 100 & 126
\end{tabular}

CUADRO 15

Distribución de los casos analizados según resolución dictada y según sexo.

\begin{tabular}{lccccc}
\hline \multirow{2}{*}{ Sexo } & \multicolumn{2}{c}{$\begin{array}{c}\text { En contra del } \\
\text { estudiante }\end{array}$} & \multicolumn{2}{c}{$\begin{array}{l}\text { A favor del } \\
\text { estudiante }\end{array}$} & \multirow{2}{*}{ Total } \\
\cline { 2 - 4 } & $\mathbf{N}^{\mathbf{0}}$ & $\%$ & $\mathbf{N}^{\circ}$ & $\%$ & \\
\hline Mujer & 104 & 65 & 37 & 65 & 141 \\
Hombre & 55 & 35 & 20 & 35 & 75 \\
Total & 159 & 100 & 57 & 100 & $216^{*}$
\end{tabular}

* No se registra información en el expediente de tres de los casos incluidos dentro de los 159 estudiantes que constituyen la población en estudio.

De los 219 estudiantes inculpados de DA, (según una consulta realizada a la base de datos institucional AS400), hasta marzo del $2011,77 \%$ se encuentra incorporado en el sistema como estudiante regular.

\section{DISCUSIÓN}

Los resultados del estudio aportan evidencia para asociar, de algún modo, la DA con el bajo rendimiento académico. Tal información es consecuente con los estudios de factores individuales sobre fraude académico llevados a cabo por Anderman et al., 1998; David et al., 1992; citado por Fernández y Ordóñez (2004).

Para este estudio se descarta, como posibles variables explicativas de la DA, el sexo, la edad, la condición de becario y la carga académica matriculada por el estudiante. En el último caso, pese a que la carga típica del estudiante infractor es de dos asignaturas por cuatrimestre podría existir alguna incidencia, ya no por el número de asignaturas matriculadas, sino por la exigencia académica que demanda cada una de ellas.

Según la literatura especializada en el tema, algunas modalidades de DA son parte de una conducta culturalmente aprehendida durante las primeras fases de escolarización. En ese sentido, Ordoñez et al. 2006 señalan que "las creencias estudiantiles sobre lo académico se han formado desde su experiencia escolar, probablemente, pero se ven reforzadas y confirmadas en la universidad y pueden estar constituyendo toda una cultura, un conjunto de significados compartidos que hace que sea posible que el fraude ocurra como parte natural de la actividad del estudiante universitario." (p. 7)

De igual forma, en el imaginario del estudiantado se ha establecido la idea de que el estudio se limita a prepararse, casi exclusivamente, para resolver instrumentos de evaluación de los aprendizajes. Spitzer, 2005 señala que los docentes usualmente declaramos "non scholae, ded vitae" (no se aprende para la escuela, sino para la vida), pero luego practicamos algo totalmente distinto: se explica 'materia' que luego se examina, para pasar a la siguiente 'materia'. Esa actitud pragmática ante el aprender y el conocer restringe, de alguna manera, las aspiraciones del estudiante porque privilegia los resultados sobre los procesos. Eso deriva en lo que Langer, 2007 califica como automatismo: "si algo se presenta como una verdad aceptada, ni siquiera surgen otras maneras aceptadas para pensar ese hecho" (p. 55). 
Por otra parte, el desarrollo de las tecnologías de la información y la comunicación (TIC) y los procesos de globalización no garantizan por sí mismos la democratización de los conocimientos. Debido a que potenciales beneficios acarreados por el desarrollo de las TIC encuentran sus límites en las capacidades individuales para validar las informaciones y construir conocimientos a partir de esa base. En congruencia con el anterior planteamiento, Pozo, 2006 afirma: "apropiarse de esa cultura simbólica que demanda la sociedad del conocimiento exige por tanto nuevas formas de adquirir conocimientos. $Y$ es que de hecho entre los conocimientos culturales que esos procesos permiten aprender está incluida o implica a su vez una cultura del aprendizaje, que entre sus muchas herramientas multimedias incluye un kit de supervivencia cognitiva compuesto por nuevos procesos de adquisición de conocimientos..." (p. 15). En ese contexto cabe preguntarse: ¿poseen los estudiantes de la UNED el "kit de supervivencia cognitiva"? O bien, ¿los procesos formativos que se desarrollan en la universidad están contemplando el fortalecimiento de ese "kit de supervivencia cognitiva" en los estudiantes?

\section{REFERENCIAS}

Comas, R. \& Sureda, J. (2007). Ciberplagio académico. Una aproximación al estado de los conocimientos. Revista Textos de la Cibersociedad, 10: 1577-3760. (Recuperado de http:// www.cibersociedad.net).

Fernández, J. \& Ordóñez, C. (2004). El fraude académico en la Universidad de los Andes ¿qué?, ¿qué tanto? y ¿por qué? Revista de Estudios Sociales, 18, 369-375.
García, E. \& Manchado, B. (s.f.). Un modelo econométrico del fraude académico en una universidad española. Recuperado de http://www.ucm.es/BUCM/cee/doc9820.html

Langer, E. (2007). Mindfulness: La conciencia plena. Barcelona, España: PAIDÓS.

Mejía, J.F. \& Ordóñez, C.L. (2004). El fraude académico en la universidad de los Andes ¿qué, tanto y porqué?. Revista de Estudios Sociales, 18: 13-18.

Ordóñez C., Mejía, J.F. \& Castellanos, S. (2006). Percepciones estudiantiles sobre el fraude académico: hallazgos y reflexiones pedagógicas. Revista de Estudios Sociales, 2337-40 Recuperado de http://dialnet.unirioja.es/servlet/ oaiart?codigo $=2348532$

Pozo, J.I. (2006). Adquisición de conocimiento; cuando la carne se hace verbo. Madrid, España: Morata.

Sureda, J., Comas, R. \& Morey, M. (2009). Las causas del plagio Académico entre el alumnado universitaria según profesorado. Revista Iberoamericana de Educación, 50: 197-220. Recuperado de http://www.rieoei.org/rie50a10.pdf

Spitzer, M. (2005). Aprendizaje neurociencia y la escuela de la vida. Barcelona, España: Ediciones Omega.

Urbina, S., \& Comas, R. (s.f.). El ciberplagio de la universidad: análisis documental de las perspectivas del profesorado y alumnado. Recuperado de http://www.sre.urv.es/web/ edutec/CDedutec/comun-pdf/santos-urbina-ramirezciberplagio.pdf

Vaamonde, J.D. \& Omar,A. (2008). La deshonestidad académica como un constructo multidimensional. Revista Latinoamericana de Estudios Educativos, 2-4: 7-27. 
